\title{
A Class of Small Injective Modules
}

\author{
Akeel Ramadan Mehdi \\ Mathematical Department/ College of Education/ \\ Al-Qadisiyah University/ Al-Diwaniya City/ Iraq \\ Email: akeel.mehdi@qu.edu.iq
}

\begin{abstract}
:
Let $R$ be a ring. In this paper, a right $R$-module $M$ is defined to be $A S$-injective if $\operatorname{Ext}^{1}(R / K, M)=0$, for any annihilator-small right ideal $K$ of $R$. We characterize rings over which every right module is $A S$-injective. Conditions under which the class of $A S$-injective right $R$-modules $\left(A S I_{R}\right)$ is closed under quotient (resp. pure submodules, direct sums) are given. Finally, we study the definability of the class $A S I_{R}$.
\end{abstract}

Keywords: Injective module, Definable class, a-small right ideal, Pure submodule.

Mathematics subject classification: 13C11,16D50,16D10. 


\section{Introduction}

Throughout $R$ is an associative ring with identity and all modules are unitary modules. If not otherwise specified, by a module (resp. homomorphism) we will mean a right $R$ module (resp. right $R$-homomorphism). We use $R$ $\operatorname{Mod}(\operatorname{resp} . \operatorname{Mod}-R)$ to denote to the class of left (resp. right) $R$-modules. If $Y \subseteq R$, then $r(Y)=\{r \in$ $R \mid Y r=0\} \quad$ (resp. $l(Y)=\{r \in R \mid r Y=0\}$ )stands for the right (resp. left) annihilator of $Y$ in $R$. We will use $M^{*}$ to denote the character module $\operatorname{Hom}_{\mathbb{Z}}(M, \mathbb{Q} / \mathbb{Z})$ of a module $M$. Let $\mathcal{G}($ resp. $\mathcal{F}$ ) be a class of right (resp. left) $R$-modules. A pair $(\mathcal{F}, \mathcal{G})$ is called almost dual pair if the class $\mathcal{G}$ is closed under direct products and summands, and for any left $R$-module $M, M \in \mathcal{F}$ if and only if $M^{*} \in \mathcal{G}[11$, p. 66]. An exact sequence $0 \rightarrow A \stackrel{\alpha}{\rightarrow} B \stackrel{\beta}{\rightarrow} C \rightarrow 0$ of right $R$-modules is said to be pure if the sequence $0 \rightarrow \operatorname{Hom}_{R}(N, A) \rightarrow \operatorname{Hom}_{R}(N, B) \rightarrow$ $\operatorname{Hom}_{R}(N, C) \rightarrow 0$ is exact, for every finitely presented right $R$-module $N$ and we called that $\alpha(A)$ is a pure submodule of $B$ [18]. A right $R$ module $M$ is called $F P$-injective if every monomorphism $\alpha: M \rightarrow N$ is pure. A right $R$ module $M$ is called pure injective if $M$ is injective with respect to all pure short exact sequences [18]. Recall that a subclass $\mathcal{G}$ of $\operatorname{Mod}-R$ is called definable if it is closed under pure submodules, direct limits and direct products [14]. A right ideal $X$ of a ring $R$ is called small in $R$ if $X+Y \neq R$, for any proper right ideal $Y$ of $R$ [8]. A right $R$-module $M$ is called small injective if $\operatorname{Ext}^{1}(R / K, M)=0$, for any small right ideal $K$ of $R$. A right ideal $I$ of $R$ is called annihilator-small (a-small) and denoted by $I \subseteq{ }^{a} R_{R}$ if for any right ideal $K$ of $R$ with $I+K=$ $R$, then $l(K)=0[13]$.
The sum of all the annihilator-small right ideals of a ring $R$ is called the right $A S$-ideal of a ring $R$ and denoted by $A_{r}$ [13].

We refer the reader to $[1,7,8,14,18]$, for general background materials.

In section 2 of this paper, we introduce the class of $A S$-injective modules. This class of modules lies between injective modules and small injective modules. We first characterize rings over which every module is $A S$-injective. Over a commutative ring $R$, we prove the equivalence of the following statements: (1) $A_{r}=0$. (2) Every module is $A S$ injective. (3) Every principal a-small right ideal of $R$ is $A S$-injective. (4) Every simple module is $A S$ injective and $A_{r} \subseteq^{a} R_{R}$. Conditions under which the class of $A S$-injective right $R$-modules $\left(A S I_{R}\right)$ is closed under quotient are given. For instance, we prove that the following statements are equivalent: (1) The class $A S I_{R}$ is closed under quotient. (2) If $K \subseteq^{a} R$, then $K$ is projective. (3) $A S I_{R}$ contains all sums of any two $A S$-injective submodules of any module. Also, we show that the class $A S I_{R}$ is closed under pure submodules if and only if all a-small right ideals in $R$ are finitely generated if and only if all $F P$-injective modules are $A S$-injective. Finally, we give conditions such that any direct sum of modules in the class $A S I_{R}$ is also belong to $A S I_{R}$. For instance, we prove that if $A_{r} \subseteq{ }^{a} R_{R}$, then the following are equivalent. (1) $A_{r}$ is a noetherian module. (2) The class $A S I_{R}$ is closed under direct sums.

Section 3 studies the definability of the class $A S I_{R}$. It is shown that the following assertions are equivalent: (1) $A S I_{R}$ is definable. (2) The class $A S I_{R}$ is closed under pure submodules and pure homomorphic images. 
(3) Every a-small right ideal in $R$ is finitely presented. (4) A module $M \in A S I_{R}$ if and only if $M^{* *} \in A S I_{R}$. Finally, we prove that if the class $A S I_{R}$ is a definable, then the following are equivalent. (1) The class of flat left $R$-modules and the class $\left\{M \in R-\operatorname{Mod} \mid M^{*}=\operatorname{Hom}_{\mathbb{Z}}(M, \mathbb{Q} / \mathbb{Z}) \in A S I_{R}\right\} \quad$ are coincide. (2) Each module in $A S I_{R}$ is $F P$-injective. (3) Each pure-injective module in $A S I_{R}$ is injective.

\section{2. $A S$-Injective modules}

Definition 2.1. A module $M$ is said to be annihilator-small injective (shortly, $A S$ injective), if $\operatorname{Ext}^{1}(R / K, M)=0$, for any annihilator-small right ideal $K$ of $R$; equivalently, if $K$ is any annihilator-small right ideal in $R$, then any $R$-homomorphism $f: K \rightarrow M$ extends to $R_{R}$. A ring $R$ is said to be right $A S$-injective if $R_{R}$ is $A S$-injective.

We will use $A S I_{R}$ to denote to the class of $A S$ injective right $R$-modules.

\section{Examples 2.2.}

(1) It is clear that $A S$-injectivity implies small injectivity, but $\mathbb{Z}$ is a small injective $\mathbb{Z}$-module [17] and clearly, it is not $A S$-injective. Thus the class of small injective modules contains properly the class of $A S$-injective modules.

(2) All injective modules are $A S$-injective and generally the converse is not true, for example, let $\left\{F_{i}\right\}_{i \in I}$ be a family of fields and let $R=$ $\prod_{i \in \Lambda} F_{i}$ be the ring product of $F_{i}$, for all $i \in \Lambda$, where addition and multiplication are define componentwise and let $K=\bigoplus_{i \in \Lambda} F_{i}$. If $\Lambda$ is infinite, then $K_{R}$ is not itself injective by [8, p. 140], but $K_{R}$ is

$A S$-injective, since $A_{r}=0$. Therefore, $A S$-injective module is a proper generalization of injective modules.
Hence $I n j_{R} \subsetneq A S I_{R} \subsetneq S I_{R}$, where $I n j_{R}$ (resp. $S I_{R}$ ) is the class of injective (resp. small injective) right $R$-modules.

\section{Remarks 2.3.}

(1) The two classes $I n j_{R}$ and $A S I_{R}$ are coinciding, when $R$ is an integral domain, since all proper right ideals are a-small in any integral domain.

(2) All finitely generated $\mathbb{Z}$-modules are not $A S$ injective and this follows from (1) and the fact that every non-trivial finitely generated $\mathbb{Z}$ module is not injective [7, p.31]. Also, we have from [17, Theorem 2.8] that any $\mathbb{Z}$-module is small injective.

(3) From (1) and [9, p.410], we have that any ring $R$ is a field if and only if it is an $A S$-injective integral domain.

Proposition 2.4. The class of $A S$-injective modules $\left(A S I_{R}\right)$ is closed under direct summands, direct products and isomorphic copies.

Proof. Clear.

Theorem 2.5. Consider the following conditions for a ring $R$.

(1) $A_{r}=0$.

(2) All modules are $A S$-injective.

(3) All principal a-small right ideals of $R$ are $A S$ injective.

(4) All principal a-small right ideals of $R$ are direct summand in $R_{R}$.

(5) All simple modules are $A S$-injective and $A_{r} \subseteq{ }^{a} R_{R}$

Then (1) and (5) are equivalent and (1) $\Longrightarrow(2) \Longrightarrow$ (3) $\Rightarrow$ (4). Moreover, if $R$ is commutative, then (4) implies (1). 
Proof. $(1) \Rightarrow(2) \Rightarrow(3)$ and

(1) $\Rightarrow$

(5) are obvious.

(3) $\Rightarrow$ (4). Let $a R \subseteq^{a} R$, where $a \in R$. By hypothesis, $a R$ is $A S$-injective and so there is a homomorphism $\alpha: R \rightarrow a R$ such that $\alpha i=I_{a R}$, where $I_{a R}: a R \rightarrow a R$ is the identity homomorphism and $i: a R \rightarrow R$ is the inclusion mapping. Thus $a R$ is a direct summand in $R_{R}$.

(4) $\Rightarrow(1)$. Let $R$ be a commutative ring. Assume that $A_{r} \neq 0$, thus there is $(0 \neq) a \in A_{r}$. By hypothesis, $A_{r} \subseteq{ }^{a} R_{R}$ and hence Lemma 1 in [13] implies $a R \subseteq \subseteq^{a} R_{R}$. By hypothesis, $a R$ is a direct summand in $R_{R}$ and hence there exist a right ideal $K$ with $a R \oplus K=R_{R}$. Since $a R \subseteq^{a} R_{R}, r(K)=0$. Since $a R+K=R$, we have $r(a R \cap K)=r(a R)+$ $r(K)$ and hence $r(a R)=R$. Thus $a R=0$, a contradiction. Therefore, $A_{r}=0$.

(5) $\Rightarrow$ (1)Assume that $A_{r} \neq 0$, thus there is $(0 \neq) a \in A_{r}$. If $A_{r}+r(a) \neq R$, then $A_{r}+r(a) \subseteq$ $C$, for some maximal right ideal $C$ of $R$. Thus $R / C$ is a simple right $R$-module. By hypothesis, $R / C$ is an $A S$-injective module. Define $\alpha: a R \rightarrow R / C$ by $\alpha(a r)=r+C$. Clearly, $\alpha$ is a well-defined right $R$-homomorphism. By $A S$ injectivity, there exist $b \in R$ with $1+C=b a+C$ and hence $1-b a \in C$. Since $a \in A_{r}$ and $A_{r}$ is a two sided ideal (by [13, Theorem 9 (1)]), we have $b a \in C$. Thus $C=R$, a contradiction. Therefore, $A_{r}+r(a)=R . \quad$ Since $A_{r} \subseteq^{a} R_{R}$ (by hypothesis), $l(r(a))=0$, so that $r(l(r(a)))=R$. By [1, Proposition 2.15, p.37], $r(a)=R$ and hence $a=0, \quad$ a contradiction. Thus $A_{r}=0$.

Recall that a ring $R$ is called regular if for any $x \in R$, there is an element $y \in R$ such that $x=x y x$ [8]
Corollary 2.6. If $R$ is a commutative regular ring, then every module is $A S$-injective and $A_{r}=0$.

Proof. By [8, Theorem 10.4.9, p. 262] and

Theorem 2.5.

It is not true in general that if $K \subseteq \subseteq^{a} R_{R}$, then $K$ is a projective right $R$-module, for example, if $R=Z_{4}$ and $K=2 R$, then $K \subseteq^{a} R_{R}$ but it is not projective right $R$-module.

Theorem 2.7. For a ring $R$, the following are equivalent.

(1) If $K \subseteq^{a} R_{R}$, then $K$ is projective.

(2) The class $A S I_{R}$ is closed under quotient.

(3) $A S I_{R}$ contains all quotients of injective modules.

(4) $A S I_{R}$ contains all sums of any two $A S-$ injective submodules of any module.

(5) $A S I_{R}$ contains all sums of any two injective submodules of any module.

Proof. (2) $\Rightarrow(3)$ and (4) $\Rightarrow(5)$ are obvious. (1) $\Longrightarrow(2)$ Let $\alpha: N \rightarrow M$ be any epimorphism, where $N$ is an $A S$-injective module and $M$ is any module. Let $\lambda: K \rightarrow M$ be any homomorphism, where $K \subseteq{ }^{a} R_{R}$. By hypothesis, $K$ is projective and hence there is a homomorphism $\beta: K \rightarrow N$ such that $\alpha \beta=\lambda$.

By $A S$-injectivity of $N$, there is a homomorphism $\gamma: R \rightarrow N$ with $\gamma i=\beta$, where $i: K \rightarrow R$ is the inclusion mapping. Put $\varphi=$ $\alpha \gamma: R \rightarrow M$, so that $\varphi i=\alpha \gamma i=\alpha \beta=\lambda$ and hence $M$ is an $A S$-injective module.

(3) $\Rightarrow$ (1) Let $K \subseteq^{a} R_{R}$. Let $\alpha: E \rightarrow N$ be an epimorphism (where $E$ is an injective module) and $\beta: K \rightarrow N$ a homomorphism. By hypothesis, $N \in A S I_{R}$ and hence there is a homomorphism $\lambda: R \rightarrow N$ with $\lambda i=\beta$, where $i: K \rightarrow R$ is the inclusion mapping. By projectivity of $R_{R}$, there is a 
homomorphism $\gamma: R \rightarrow E$ such that $\alpha \gamma=\lambda$. Let $\tilde{\alpha}: K \rightarrow E$ be the restriction of $\gamma$ over $K$. Clearly, $\alpha \tilde{\alpha}=\beta$ and hence from Proposition 5.2.10 in [2, p.148] we get that $K$ is projective.

(2) $\Rightarrow$ (4) Let $M_{1}$ and $M_{2}$ be two

injective submodules of module $M$. By Proposition 2.4, $\quad M_{1} \oplus M_{2} \in A S I_{R}$. Since $M_{1}+M_{2}$ is a homomorphic image of $M_{1} \oplus M_{2}$, we have $M_{1}+M_{2} \in A S I_{R}$, by hypothesis.

$(5) \Rightarrow(3)$. By similar argument as in the proof of Theorem $2.14((6) \Rightarrow(3))$ in [12].

Proposition 2.8. For a ring $R$, consider the following conditions.

(1) Every module is $A S$-injective.

(2) $R_{R}$ is $A S$-injective and the class $A S I_{R}$ is closed under quotient.

(3) For any $x \in R$, if $x R \subseteq^{a} R_{R}$, then there is $y \in R$ such that $x=x y x$.

Then $(1) \Rightarrow(2) \Rightarrow$ (3) and if $R$ is commutative, then (3) implies (1).

Proof. (1) $\Rightarrow$ (2). Clear.

(2) $\Rightarrow$ (3). Let $x \in R$ such that $x R \subseteq^{a} R_{R}$. Since $A S I_{R}$ is closed under quotient (by hypothesis), $x R$ is projective, by Theorem 2.7. Define $\alpha: R \rightarrow x R$ by $\alpha(r)=x r$, for all $r \in R$. Clearly, $\alpha$ is an epimorphism, so that there is a homomorphism $f: x R \rightarrow R$ with $\alpha f(a)=a$, for all $a \in x R$. Since $R_{R}$ is $A S$-injective (by hypothesis), there is a homomorphism $g: R \rightarrow R$ such that $g i=f$, where $i: x R \rightarrow R$ is the inclusion mapping. Thus $x=$ $\alpha(f(x))=\alpha(g(x))=x y x$, where $y=g(1) \in R$. (3) $\Rightarrow(1)$. Suppose that $R$ is a commutative ring. Let $x R \subseteq^{a} R_{R}$, where $x \in R$. By hypothesis, there is $y \in R$ with $x=x y x$. Let $e=x y$. Clearly, $e$ is an idempotent of $R$ and $x R=e R$, so that $x R$ is a direct summand of $R_{R}$. Therefore, the result follows by Theorem 2.5.
Proposition 2.9. For a ring $R$, the following are equivalent.

(1) All a-small right ideals in $R$ are finitely generated.

(2) The class $A S I_{R}$ is closed under pure submodules.

(3) All FP-injective modules are $A S$ injective.

Proof. (1) $\Rightarrow$ (2). Let $M \in A S I_{R}$ and $K$ a pure submodule of $M$. Let $I \subseteq{ }^{a} R_{R}$, thus the hypothesis implies that $I$ is finitely generated and so $R / I$ is a finitely presented. Hence the sequence $\operatorname{Hom}_{R}(R / I, M) \rightarrow$ $\operatorname{Hom}_{R}(R / I, M / K) \rightarrow 0$ is exact. By [6, Theorem XII.4.4 (4), p. 491], the exact sequence $\operatorname{Hom}_{R}(R / I, M) \rightarrow \operatorname{Hom}_{R}(R / I, M / K) \rightarrow$ $\operatorname{Ext}^{1}(R / I, K) \rightarrow \operatorname{Ext}^{1}(R / I, M) \quad$ and $\quad$ so $\operatorname{Ext}^{1}(R / I, K)=0$. Thus, $K \in A S I_{R}$ and hence the class $A S I_{R}$ is closed under pure submodules.

(2) $\Longrightarrow(3)$. If $M$ is any $F P$-injective module, then $M$ is a pure submodule of an $A S$-injective module. By hypothesis, $M \in A S I_{R}$.

(3) $\Rightarrow(1)$. Let $\quad I \subseteq^{a} R_{R} \quad$ and $\quad \alpha: I \rightarrow M \quad$ a homomorphism, where $M$ is an FPinjective module. By hypothesis, $M$ is $A S$-injective and hence $\alpha$ extends to $R_{R}$. By [4], $I$ is finitely generated.

Corollary 2.10. If each a-small right ideal in a ring $R$ is finitely generated, then the class $A S I_{R}$ is closed under direct sums.

Proof. Let $\left\{M_{i} \mid i \in I\right\}$ be a subclass of $A S I_{R}$. By Proposition 2.4, $\prod_{i \in I} M_{i} \in A S I_{R}$. By [14, Proposition 2.1.10, p. 57], $\bigoplus_{i \in I} M_{i}$ is a pure submodule in $\prod_{i \in I} M_{i}$ and hence $\bigoplus_{i \in I} M_{i} \in A S I_{R}$, by Proposition 2.9 . 
Theorem 2.11. For a ring $R$, consider the following conditions.

(1) $A_{r}$ is a noetherian module.

(2) The class $A S I_{R}$ is closed under direct sums.

(3) $M^{\mathbb{N}}$ is $A S$-injective, for any $A S-$ injective module $M_{R}$

(4) $M^{\mathbb{N}}$ is $A S$-injective, for any injective module $M_{R}$.

Then (1) $\Longrightarrow(2) \Longrightarrow(3) \Longrightarrow$ (4) and if $A_{r} \subseteq^{a} R_{R}$, then $(4) \Rightarrow(1)$.

Proof. (2) $\Rightarrow$ (3) and (3) $\Rightarrow$ (4) are clear.

$(1) \Rightarrow(2)$. By [13, Theorem 9(1)] and Corollary 2.10 .

(4) $\Rightarrow$ (1). Let $A_{r} \subseteq^{a} R_{R}$ and let $K_{1} \subseteq K_{2} \subseteq \cdots$ be a chain of right ideals of $R$ with $K_{i} \subseteq A_{r}$. Let $E=\bigoplus_{i=1}^{\infty} E_{i}$, where $E_{i}=E\left(R / K_{i}\right)$. For every $i \geq 1$, put $M_{i}=\prod_{j=1}^{\infty} E_{j}=E_{i} \oplus\left(\prod_{\substack{j=1 \\ j \neq i}}^{\infty} E_{j}\right)$, thus $M_{i}$ is injective. By hypothesis, $\quad \bigoplus_{i=1}^{\infty} M_{i}=$ $\left(\bigoplus_{i=1}^{\infty} E_{i}\right) \oplus\left(\bigoplus_{i=1}^{\infty} \prod_{\substack{j \neq 1 \\ j \neq i}}^{\infty} E_{j}\right)$ is $A S$-injective. By Proposition 2.4, $E$ is $A S$-injective. Define $\alpha: \bigcup_{i=1}^{\infty} K_{i} \rightarrow E$ by $\alpha(x)=\left(x+K_{i}\right)_{i}$. Clearly, $\alpha$ is a well-defined homomorphism. By hypothesis, $A_{r} \subseteq^{a} R_{R}$ and hence Lemma 1 in [13] implies that $\bigcup_{i=1}^{\infty} K_{i} \subseteq^{a} R_{R}$. Thus $\alpha$ extends to a homomorphism $\beta: R \rightarrow E$ and hence $\beta(R) \subseteq \bigoplus_{i=1}^{n} E\left(R / K_{i}\right)$ for some $n \in \mathbb{N}$, since $R$ is finitely generated. Then $\alpha\left(\bigcup_{i=1}^{\infty} K_{i}\right) \subseteq \bigoplus_{j=1}^{n} E\left(R / K_{i}\right)$.

So, if $a \in \cup_{i=1}^{\infty} K_{i}$, then $a \in K_{m}$ for all $m>n$, and hence $\cup_{i=1}^{\infty} K_{i}=K_{n+1}$. Therefore, the chain $K_{1} \subseteq$ $K_{2} \subseteq \cdots$ terminates at $K_{n+1}$ and hence $A_{r}$ is a noetherian module.

Corollary 2.12. If $A_{r} \subseteq{ }^{a} R_{R}$, then the following are equivalent.

(1) $A_{r}$ is a noetherian module.

(2) Direct sum of injective modules is $A S-$ injective.
Lemma 2.13. If $R$ satisfies ACC (ascending chain condition) on a-small right ideals of $R$, then $A_{r} \subseteq^{a} R_{R}$

Proof. Let $\mathcal{H}=\left\{K \mid K \subseteq^{a} R_{R}\right\}$. Thus $\mathcal{H}$ has a maximal element, say $N$ (by Zorn's lemma). Since $A_{r}=\sum_{K \in \mathcal{H}} K$, it follows that $A_{r}=N$ and so $A_{r} \subseteq^{a} R_{R}$.

Proposition 2.14. For a ring $R$, the following are equivalent.

(1) $R$ satisfies ACC on a-small right ideals.

(2) $A_{r}$ is a noetherian $R$-module.

(3) $M^{\mathbb{N}}$ is $A S$-injective, for any injective module $M_{R}$ and $A_{r} \subseteq^{a} R_{R}$.

Proof. (1) $\Rightarrow$ (2). Let $N_{1} \subseteq N_{2} \subseteq \cdots$ be a chain of right ideals of $R$ in $A_{r}$. By Lemma 2.13, $A_{r} \subseteq^{a} R_{R}$. By [13, Lemma 1], $N_{i}$ are a-small right ideals. By hypothesis, the chain $N_{1} \subseteq N_{2} \subseteq \cdots$ terminates and hence $A_{r}$ is a noetherian $R$-module.

(2) $\Rightarrow(1)$. By [13, Theorem 9(1)].

(2) $\Rightarrow(3)$. By Theorem 2.11 and Lemma 2.13 .

(3) $\Rightarrow(2)$. By Theorem 2.11.

\section{Definability of the class $A S I_{R}$}

For any class $\mathcal{G}$ of right $R$-modules, we will set $\mathcal{G}^{+}=\{M \in \operatorname{Mod}-R \mid M$ is a pure submodule of a module in $\mathcal{G}\}$ and $\mathcal{G}^{\ominus}=\left\{M \in R\right.$-Mod $\mid M^{*}=$ $\left.\operatorname{Hom}_{\mathbb{Z}}(M, \mathbb{Q} / \mathbb{Z}) \in \mathcal{G}\right\}$.

Proposition 3.1. The pair $\left(\left(A S I_{R}\right)^{\ominus}, A S I_{R}\right)$ is an almost dual pair over a ring $R$.

Proof. By proposition 2.4, the class $A S I_{R}$ is closed under direct summands and direct products. By [11, Proposition 4.2.11, p.72], the pair $\left(\left(A S I_{R}\right)^{\ominus}, A S I_{R}\right)$ is an almost dual pair over a ring $R$. 
Corollary 3.2. Consider the following conditions for the class $A S I_{R}$ over a ring $R$.

(1) The class $A S I_{R}$ is definable.

(2) $\left(A S I_{R},\left(A S I_{R}\right)^{\ominus}\right)$ is an almost dual pair over a ring $R$.

(3) $\left(A S I_{R}\right)^{*} \subseteq\left(A S I_{R}\right)^{\ominus}$.

(4) $\left(A S I_{R}\right)^{* *} \subseteq A S I_{R}$.

(5) The class $A S I_{R}$ is closed under pure homomorphic images.

Then (1) $\Leftrightarrow(2),(1) \Rightarrow(3), \quad(1) \Rightarrow$ (5) and (3) $\Leftrightarrow$ (4). Moreover, if all a-small right ideals in $R$ are finitely generated, then all five conditions are equivalent.

Proof. (1) $\Leftrightarrow$ (2). By Proposition 3.1 and [11, Proposition 4.3.8, p. 89].

(1) $\Rightarrow$ (3). Since $A S I_{R}$ is a definable class, it is closed under pure submodules and hence $\left(A S I_{R}\right)^{+}=$ $A S I_{R}$. Since $\left(\left(A S I_{R}\right)^{\ominus}, A S I_{R}\right)$ is an almost dual (by Proposition 3.1), it follows from [11, Theorem 4.3.2, p.85], that $\left(A S I_{R}\right)^{*} \subseteq\left(A S I_{R}\right)^{\ominus}$.

(1) $\Rightarrow$ (5). By [14, 3.4.8, p. 109].

(3) $\Leftrightarrow$ (4). By Proposition 3.1 and [11, Theorem 4.3.2, p.85].

(4) $\Rightarrow$ (1) and (5) $\Rightarrow$ (1). Suppose that all a-small right ideals in $R$ are finitely generated. By Proposition 2.9, the class $A S I_{R}$ is closed under pure submodules and hence $\left(A S I_{R}\right)^{+}=A S I_{R}$. Thus the results follow from [11, Theorem 4.3.2, p.85].

Corollary 3.3. If every $A S$-injective modules is pure-injective, then the following statements are equivalent for a class $A S I_{R}$ over a ring $R$.

(1) $A S I_{R}$ is definable.

(2) $A S I_{R}$ is closed under direct sums.

(3) $\left(A S I_{R}\right)^{+}=A S I_{R}$.

(4) Each a-small right ideal in $R$ is finitely generated.
Proof. The equivalence of (1), (2) and (3) follows from Proposition 3.1 and [11, Theorem 4.5.1, p.103].

(1) $\Leftrightarrow$ (4). By Proposition 3.1, Proposition 2.9 and [11, Theorem 4.5.1, p.103].

Lemma 3.4. A left $R$-module $M \in\left(A S I_{R}\right)^{\ominus}$ if and only if $\operatorname{Tor}_{1}(R / I, M)=0$, for any a-small right ideal $I$ of a ring $R$.

Proof. Let $M$ be a left $R$-module and $I \subseteq^{a} R_{R}$. By [5, Theorem 3.2.1, p.75], $\operatorname{Ext}^{1}(R /$ $\left.I, M^{*}\right) \cong\left(\operatorname{Tor}_{1}(R / I, M)\right)^{*}$, so that $\operatorname{Tor}_{1}(R / I, M)=$ 0 if and only if $M^{*} \in A S I_{R}$. Hence $\left({ }_{R} A S F, A S I_{R}\right)$ is an almost dual, where ${ }_{R} A S F=\{M \in R$-Mod $\mid$ $\operatorname{Tor}_{1}(R / I, M)=0$, for any a-small right ideal $I$ of $R\}$. By $[11, \quad$ Proposition 4.2.11, p.72], $\left(A S I_{R}\right)^{\ominus}={ }_{R} A S F$.

A right $R$-module $M$ is called $n$-presented if there is an exact sequence $F_{n} \rightarrow F_{n-1} \rightarrow \cdots \rightarrow F_{0} \rightarrow$ $M \rightarrow 0$, with each $F_{i}$ is a finitely generated free right $R$-modules [3].

Theorem 3.5. The following statements are equivalent for a class $A S I_{R}$ over a ring $R$.

(1) $A S I_{R}$ is definable.

(2) The class $A S I_{R}$ is closed under pure submodules and pure homomorphic images.

(3) Every a-small right ideal in $R$ is finitely presented.

(4) A module $M \in A S I_{R}$ if and only if $M^{*} \in$ $\left(A S I_{R}\right)^{\ominus}$.

(5) A module $M \in A S I_{R}$ if and only if $M^{* *} \in A S I_{R}$.

Proof. (1) $\Rightarrow$ (2). By [14, 3.4.8, p. 109].

(2) $\Rightarrow$ (3). Let $M$ be any $F P$-injective module, thus there is a pure exact sequence $0 \rightarrow M \stackrel{i}{\rightarrow} E$ $\stackrel{\pi}{\rightarrow} E / M \rightarrow 0$, where $E$ is an injective right $R$ module. By hypothesis, $E / M \in A S I_{R}$. 
Let $K \subseteq{ }^{a} R_{R}$, thus $\operatorname{Ext}^{1}(R / K, E / M)=0$. By $[6$, Theorem 4.4 (4), p. 491], the sequence $0=$ $\operatorname{Ext}^{1}(R / K, E / M) \rightarrow \operatorname{Ext}^{2}(R / K, M) \rightarrow$

$\operatorname{Ext}^{2}(R / K, E)=0$ is exact and hence $\operatorname{Ext}^{2}(R / K, M)=0$. By [13, Theorem 4.4 (3), p. 491], the sequence

$0=\operatorname{Ext}^{1}(R, M) \rightarrow \operatorname{Ext}^{1}(K, M) \rightarrow$

$\operatorname{Ext}^{2}(R / K, M)=0 \quad$ is exact, so that $\operatorname{Ext}^{1}(K, M)=0$. By hypothesis, $A S I_{R}$ is closed under pure submodules, so that $K$ is finitely generated by Proposition 2.9 and hence [4, Proposition, p. 361] implies that $K$ is finitely presented.

(3) $\Longrightarrow$ (1). Let $M \in A S I_{R}$. Let $K \subseteq^{a} R_{R}$, thus $K$ is finitely presented (by hypothesis) and hence there is an exact sequence $F_{2} \stackrel{\alpha_{2}}{\longrightarrow} F_{1} \stackrel{\alpha_{1}}{\longrightarrow} K \rightarrow 0$, where $F_{1}, F_{2}$ are finitely generated free right $R-$ modules. Let $\beta=i \alpha_{1}$, where $i: K \rightarrow R$ is the inclusion mapping, thus the sequence $F_{2} \stackrel{\alpha_{2}}{\longrightarrow} F_{1}$ $\stackrel{\beta}{\longrightarrow} R \stackrel{\pi}{\longrightarrow} R / K \rightarrow 0$ is exact, where $\pi: R \rightarrow R / K$ is the natural epimorphism. Hence $R / K$ is a 2-presented module, so that from [3, Lemma 2.7 (2)] we have $\operatorname{Tor}_{1}\left(R / K, M^{*}\right) \cong\left(\operatorname{Ext}^{1}(R / K, M)\right)^{*}=0$. By Lemma 3.4, $M^{*} \in\left(A S I_{R}\right)^{\ominus}$ and hence $\left(A S I_{R}\right)^{*} \subseteq\left(A S I_{R}\right)^{\ominus}$. By hypothesis, every a-small right ideal in $R$ is finitely generated, so that $A S I_{R}$ is closed under pure submodules by Proposition 2.9. By Theorem 3.2, $A S I_{R}$ is a definable class.

$(1) \Rightarrow(4)$. By Corollary 3.2, $\left(A S I_{R},\left(A S I_{R}\right)^{\ominus}\right)$ is an almost dual pair and hence a module $M \in A S I_{R}$ if and only if $M^{*} \in\left(A S I_{R}\right)^{\ominus}$.

(4) $\Rightarrow$ (5). By hypothesis, $\left(A S I_{R}\right)^{*} \subseteq\left(A S I_{R}\right)^{\ominus}$. By Corollary 3.2, $\left(A S I_{R}\right)^{* *} \subseteq A S I_{R}$. Hence for any right $R$-module $M$, if $M \in A S I_{R}$, then $M^{* *} \in A S I_{R}$.

Conversely, if $M^{* *} \in A S I_{R}$, then $M^{*} \in\left(A S I_{R}\right)^{\ominus}$. By hypothesis, $M \in A S I_{R}$.
(5) $\Rightarrow(1)$. Let $N$ be a $F P$-injective module, thus there is a pure exact sequence $0 \rightarrow N \rightarrow E$ $\rightarrow E / N \rightarrow 0$, where $E$ is an injective right $R$ module. By [18, 34.5, p.286], the sequence $0 \rightarrow N^{* *}$ $\rightarrow E^{* *} \rightarrow(E / N)^{* *} \rightarrow 0$ is split. By hypothesis, $E^{* *} \in A S I_{R}$ and hence $N^{* *} \in A S I_{R}$. By hypothesis, $N \in A S I_{R}$ so that $A S I_{R}$ is closed under pure submodules by Proposition 2.9. Thus $A S I_{R}$ is definable class by Corollary 3.2.

Note that if the class $A S I_{R}$ is closed under pure submodules, then $\left(A S I_{R}\right)^{+}=A S I_{R}$. Thus we have the following corollary.

Corollary 3.6. The class $A S I_{R}$ is a definable if and only if it is closed under pure submodules and the class $\left(A S I_{R}\right)^{+}$is a definable.

Corollary 3.7. If the class $A S I_{R}$ is a definable, then the following are equivalent.

(1) The class of flat left $R$-modules and the class $\left(A S I_{R}\right)^{\ominus}$ are coincide.

(2) Every module in $A S I_{R}$ is $F P$-injective.

(3) Every pure-injective module in $A S I_{R}$ is injective.

Proof. $(1) \Rightarrow(2)$. Let $M \in A S I_{R}$, thus $M^{*} \in$ $\left(A S I_{R}\right)^{\ominus}$ by Corollary 3.2. By hypothesis, $M^{*}$ is a flat left $R$-module and hence Proposition 3.54 in $[15, \mathrm{p} .136] \quad$ implies that $M^{* *}$ is injective. Since $M$ is a pure submodule in $M^{* *}$, we have $M$ is

$F P$-injective by $[18,35.8$, p.301].

(2) $\Rightarrow$ (3). Let $M$ be any pure-injective module in $A S I_{R}$. Let $\mathcal{E}: 0 \rightarrow M \rightarrow M^{\prime} \rightarrow M^{\prime \prime} \rightarrow 0$ be any exact sequence. By hypothesis, $M$ is $F P$-injective. By [16, Proposition 2.6], the sequence $\mathcal{E}$ is pure and hence pure-injectivity of $M$ implies that the sequence $\mathcal{E}$ is split by [18, 33.7, p. 279] . Therefore, $M$ is injective. 
(3) $\Rightarrow(1)$. Let $M$ be a flat left $R$-module, thus $\operatorname{Tor}_{1}(N, M)=0$, for any right $R-$ module $N$. By Lemma 3.4, $M \in\left(A S I_{R}\right)^{\ominus}$. Conversely, if $M \in\left(A S I_{R}\right)^{\ominus}$, then $M^{*} \in A S I_{R}$. By [14, Proposition 4.3.29, p. 149], $M^{*}$ is a pure injective module. By hypothesis, $M^{*}$ is injective and hence $M$ is flat by [10, Theorem, p.239].

\section{References:}

[1] F. W. Anderson and K. R. Fuller, Rings and Categories of Modules, Grad. Texts in Math., Vol. 13, Springer, 1974.

[2] P. E. Bland, Rings and Their Modules, Walter de Gruyter \& Co., Berlin, 2011.

[3] J. L. Chen and N. Q. Ding, On coherent rings, Comm. Algebra 24 (1996), 32113216.

[4] E. E. Enochs, A note on absolutely pure modules, Canad. Math. Bull. 19 (1976), 361-362.

[5] E. E. Enochs and O. M. G. Jenda, Relative Homological Algebra, Walter de Gruyter, 2000.

[6] P. A. Grillet, Abstract Algebra, 2nd edition, GTM 242, Springer, 2007.

[7] P. J. Hilton and U. Stammbach, course in homological algebra, Springer-Verlag, New York, 1971.

[8] F. Kasch, Modules and Rings, Academic Press, London, New York, 1982.

[9] G. B. Klatt and L. S. Levy, Pre-self-injective rings, Tran. Amer. Math .Soc., 122 (1969), 407-419. [10] J. Lambek, A module is flat if and only if its character module is injective, Canad. Math. Bull., 7 (1964), 237-243.
[11] A. R. Mehdi, Purity relative to classes of finitely presented modules, $\mathrm{PhD}$ Thesis, Manchester University, 2013.

[12] A. R. Mehdi and A. S. Tayyah, SSInjective Modules and Rings, J. ALQadisiyah for Comp. Sci. and Math., 9 (2) 2017, 57 -70 .

[13] W. K. Nicholson and Y. Zhou, Annihilatorsmall right ideals, Algebra Colloq., 18(1) (2011), 785-800.

[14] M. Prest, Purity, Spectra and Localisation, Cambridge University Press, 2009.

[15] J. J. Rotman, An Introduction to Homological Algebra, Springer, 2009.

[16] B. Stenström, Coherent rings and FPinjective modules, J. London. Math. Soc. 2 (1970), 323-329.

[17] L.V. Thuyet and T. C. Quynh, On small injective rings and modules, J. Algebra and Its Applications 8 (2009), 379-387.

[18] R. Wisbauer, Foundations of Module and Ring Theory, Gordon and Breach, 1991. 


\title{
صنف من المقاسات الأغمارية الصغيرة
}

\author{
عقيل رمضان مهدي \\ جامعة القادية/ كلية التربية/ قشم الرياضيات
}

لتكن R حلقة. في هذا البحث المقاس الايمن MS - على الحلقة R عرف ليكون اغماري من النمط

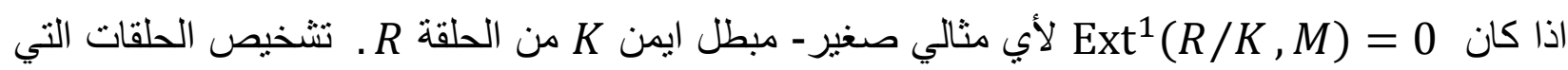
يكون كل مقاس معرف عليها هو اغماري من النمط - AS. الثروط التي بموجبها يكون صنف المقاسات

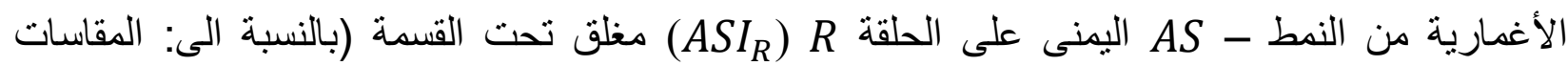
الجزئية النقية، الجمع المباثر) قد اعطيث. اخيراً، ندرس قابلية التعريف للصنف (ASI 